\author{
UNIVERSIDADE DE SÃO PAULO \\ FACULDADE DE ODONTOLOGIA DE BAURU
}

TAMIRES DAIANE DA SILVA

Aspectos psicológicos e limiar de dor em indivíduos com câncer com risco de desenvolver mucosite oral quimioinduzida 



\section{Aspectos psicológicos e limiar de dor em indivíduos com câncer com risco de desenvolver mucosite oral quimioinduzida}

Dissertação apresentada a Faculdade de Odontologia de Bauru da Universidade de São Paulo para obtenção do título de Mestre em Ciências no Programa de Ciências Odontológicas Aplicadas na área de concentração Biologia Oral, Estomatologia, Radiologia e Imaginologia.

Orientador: Prof. Dr. Paulo Sérgio da Silva Santos 
Silva, Tamires Daiane da

Aspectos psicológicos e limiar de dor em indivíduos com câncer com risco de desenvolver mucosite oral quimioinduzida / Tamires Daiane da Silva -- Bauru, 2020.

83 p. : il. ; $31 \mathrm{~cm}$.

Dissertação (Mestrado) -- Faculdade de Odontologia de Bauru, Universidade de São Paulo, 2020.

Orientador: Prof. Dr. Paulo Sérgio da Silva Santos

Autorizo, exclusivamente para fins acadêmicos e científicos, a reprodução total ou parcial desta dissertação/tese, por processos fotocopiadores e outros meios eletrônicos.

Assinatura:

Data: 
FOLHA DE APROVAÇÃO 



\section{DEDICATÓRIA}

Dedico esta dissertação aos meus pais, Maria José Pelicula da Silva e José Carlos da Silva Filho, à minha irmã Thais da Silva e à minha sobrinha Ana Clara Guimarães Monteiro, que me apoiaram e me motivaram a ingressar no mestrado e foram de fundamental importância nesta reta final.

Dedico também a todos os pacientes oncológicos que participaram da minha pesquisa, pois sem o aceite e a disponibilidade deles, seria impossível a realização deste trabalho. Muito obrigada! 



\section{AGRADECIMENTOS}

Primeiramente agradeço a Deus, porque Ele é a causa primeira de todas as coisas!

Agradeço aos meus pais, Maria e José por me apoiarem incansavelmente durante este ciclo; esta jornada não é só minha, é nossa!

Agradeço à minha irmã, Thais e ao meu cunhado Rafael que sempre se mostraram proativos, se disponibilizando a me levar de Jaú à Bauru por inúmeras vezes e a minha sobrinha, Ana Clara por sempre ir à minha casa e alegrar os meus dias.

Agradeço a Solimar Bonjardim, professora de outra instituição que pacientemente me ajudou e me motivou a ingressar no mestrado. Sem o apoio e confiança dela, eu não estaria aqui hoje.

Agradeço ao professor Leonardo Bonjardim por confiar no meu potencial e me motivar a entrar no mestrado da FOB-USP.

Agradeço imensamente ao professor Paulo Sérgio da Silva Santos por ter aceitado me orientar, mesmo sendo de uma área diferente da minha. Desde o início o professor Paulo deixou claro a dificuldade de orientar pós-graduandos de outras áreas, mas nunca desistiu de mim. Professor Paulo, o senhor é exemplo de caráter e ser-humano. Obrigada por ter me dado a oportunidade de ter trabalhado junto do senhor e da sua equipe!

Agradeço à Dalva (secretária da Biologia Oral) e ao André (técnico de laboratório da Biologia Oral), por me acolherem e me ajudarem desde o início da pós-graduação. Amo o apelido que carinhosamente eles me deram: "nossa pupila". Obrigada pelo carinho meus amigos!

Agradeço minha amiga Debora Foger, que foi a primeira pós-graduanda a me estender as mãos no início do mestrado; agradeço pela paciência e ensinamentos a respeito da confecção de artigos; agradeço pela parceria em escritas de artigos, aliás, parceria esta que renderam duas publicações. 

Agradeço às amigas Dayanne Simões e Natalia Santaella que me ensinaram muito sobre as condições acadêmicas, mas sobretudo, me ensinaram a enxergar a vida com os olhos da alma. Obrigada por me darem a oportunidade de ver a quão maravilhosa é a vida!

Agradeço à Raquel Caminha, a mãezona de todos nós pós-graduandos. Obrigada Quel pela ajuda que inúmeras vezes você me deu; obrigada por me ajudar encaminhando os participantes da pesquisa.

Agradeço à Gabriela Chicrala, grande amiga que me ensinou muito sobre mucosite oral, me deu hospedagem em sua casa várias vezes e sempre foi proativa com todos os pós-graduandos.

Agradeço aos amigos Gabriel Telles, Aneliza Moraes, Patricia Alcântara, Caroline Carvalho e Guilherme Simpione, por estarmos sempre unidos, ajudando uns aos outros.

Agradeço à professora Cassia Rubira por disponibilizar uma pós-graduanda formada em Odontologia, para me ajudar com a coleta da pesquisa.

Agradeço à Caroline Oliveira, pós-graduanda que me ajudou a coletar as informações da pesquisa, que pertenciam ao âmbito da Odontologia.

Agradeço ao Anderson, à Poliane e à Sueli, funcionários do Centro de Pesquisa Clínica, que sempre que solicitados, me ajudaram com a pesquisa.

Agradeço à professora Malú Tabaquim, neuropsicóloga, docente da FOB e do HRAC, por ter me dado a oportunidade de atuar junto da sua equipe de trabalho e pesquisa, no Laboratório de Neuropsicologia do HRAC.

O presente trabalho foi realizado com o apoio da Coordenação de Aperfeiçoamento de Pessoal de Nível Superior - Brasil (CAPES) - Código de Financiamento 001. 



\section{RESUMO}

Dentre os mais variados efeitos colaterais que resultam da quimioterapia, a complicação que aparece com mais frequência é a mucosite oral (MO). A mucosite oral (MO) é a inflamação da parte interna da boca, resultando no surgimento de dor, eritemas e/ou úlceras de difícil cicatrização. Este estudo teve como objetivo identificar quais aspectos psicológicos estão presentes em pacientes oncológicos acometidos por mucosite oral (MO) e a relação com limiar de dor. Esta pesquisa é caracterizada como um estudo piloto, de campo, transversal, que teve amostra estabelecida por conveniência; os procedimentos utilizados foram: avaliação da mucosite oral (MO) pela Organização Mundial da Saúde (OMS) e Oral Mucositis Assessment Scale (OMAS); escala visual analógica de dor (EVA); avaliação do limiar de dor por meio do algômetro analógico; teste qualitativo sensorial; escalas Beck e; depression, anxiety and stress scale-21 (DASS-21). A amostra constituiu-se de 11 indivíduos, com idade mediana de 56 anos. A distribuição de gênero foi de oito $(72,7 \%)$ mulheres e três $(27,3 \%)$ homens. Verificou-se que a incidência da mucosite oral influencia diretamente no aumento da depressão e ansiedade, quando correlacionadas. Vale ressaltar que o ponto positivo dessa pesquisa é que os 11 participantes foram avaliados em três momentos distintos e pôde-se obter resultados estatisticamente relevantes no quesito incidência da $\mathrm{MO}$, dor, depressão e ansiedade ao correlacioná-los. Acredita-se que esse estudo seja relevante, uma vez que não há trabalhos científicos na literatura que abordem a relação entre a incidência da MO, limiar de dor e aspectos psicológicos.

Palavras-chave: Oncologia. Quimioterapia combinada. Mucosite oral. PsicoOncologia. 



\section{ABSTRACT \\ Psychological aspects and pain threshold in individuals with cancer at risk of developing chemo-induced oral mucositis}

Among the most varied side effects that result from chemotherapy, the complication that appears most frequently is oral mucositis (OM). Oral mucositis (OM) is the inflammation of the inside of the mouth, resulting in the appearance of pain, erythema and / or ulcers that are difficult to heal. This study aimed to identify which psychological aspects are present in cancer patients affected by oral mucositis (OM) and the relationship with pain threshold. This research is characterized as a pilot, field, cross-sectional study, whose sample was established for convenience; the procedures used were: assessment of oral mucositis (OM) by the World Health Organization (WHO) and Oral Mucositis Assessment Scale (OMAS); visual analogue pain scale (VAS); assessment of pain threshold using an analog algometer; qualitative sensory test; Beck scales and; depression, anxiety and stress scale-21 (DASS-21). The sample consisted of 11 individuals, with a median age of 56 years. The gender distribution was eight $(72.7 \%)$ women and three $(27.3 \%)$ men. It was found that the incidence of oral mucositis directly influences the increase in depression and anxiety, when correlated. It is worth mentioning that the positive point of this research is that the 11 participants were evaluated at three different moments and it was possible to obtain statistically relevant results in terms of the incidence of $\mathrm{OM}$, pain, depression and anxiety when correlating them. This study is believed to be relevant, since there are no scientific studies in the literature that address the relationship between the incidence of $\mathrm{OM}$, pain threshold and psychological aspects.

Keywords: Medical oncology. Drug therapy, combination. Oral mucositis. PsychoOncology. 



\section{LISTA DE ILUSTRAÇÕES}

\section{- FIGURAS}

Figura 1 - Estruturação dos tempos em que foi realizada a pesquisa ..................31

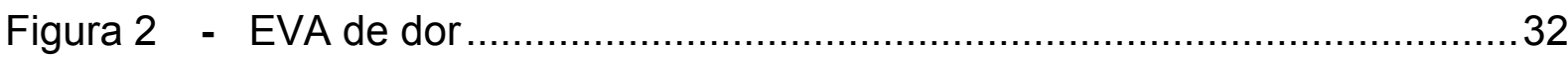

Figura 3 - Classificação das lesões de mucosite oral OMS ..............................33

Figura 4 - Classificação das lesões de mucosite oral OMAS .............................33

Figura 5 - Algômetro analógico............................................................. 34

\section{- GRÁFICOS}

Gráfico 1 - Avaliação da depressão nos três momentos da pesquisa por meio da DASS-21

Gráfico 2 - Avaliação da ansiedade nos três momentos da pesquisa por meio da DASS-21

Gráfico 3 - Presença de depressão avaliada pelo BDI (Beck Depression Inventory) no primeiro e no segundo momento da pesquisa.. 44

Gráfico 4 - Presença de ansiedade avaliada pelo BAI (Beck Anxiety Inventory) no primeiro e no segundo momento da pesquisa.

Gráfico 5 - Avaliação do limiar de dor por meio do algômetro analógico 



\section{LISTA DE TABELAS}

Tabela 1 - Variáveis demográficas de sexo, idade, religião, estado civil, escolaridade e profissão ................................................... 39

Tabela 2 - Variáveis clínicas: tipos de câncer, comorbidades e ausência de comorbidades................................................................ 40

Tabela 3 - Correlação entre mucosite oral, depressão, ansiedade e estresse (DASS-21)

Tabela 4 - Correlação entre mucosite oral, depressão, ansiedade, desesperança e ideação suicida (Escalas Beck)

Tabela 5 - Correlação entre mucosite oral e limiar de dor por meio do algômetro analógico 



\section{SUMÁRIO}

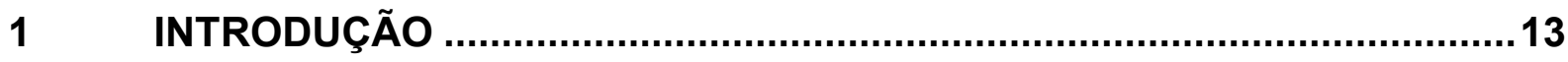

2 REVISÃO DE LITERATURA

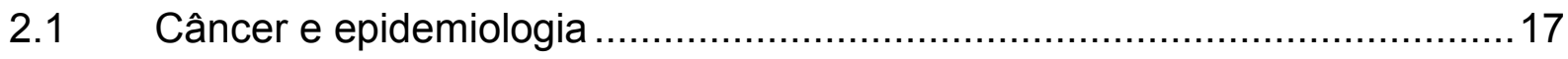

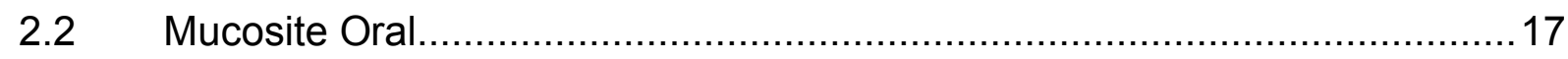

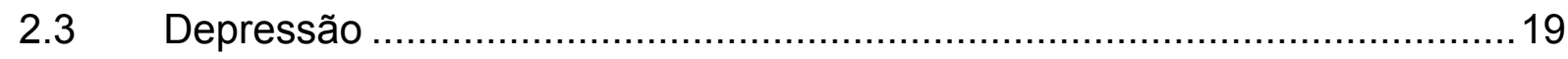

$2.4 \quad$ Ansiedade

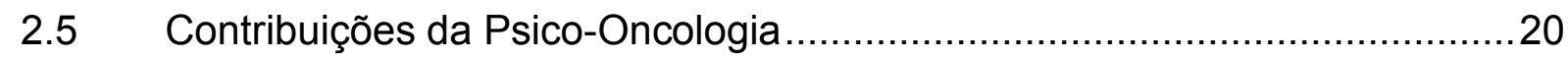

2.6 O papel da Psicologia no Atendimento Oncológico ...................................21

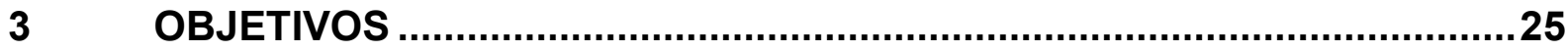

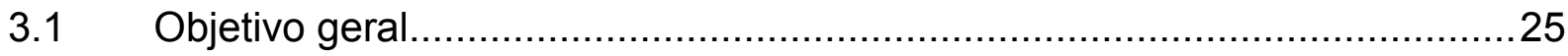

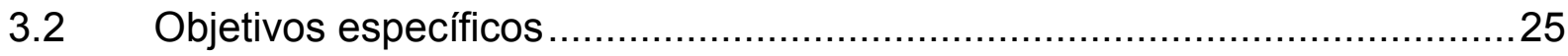

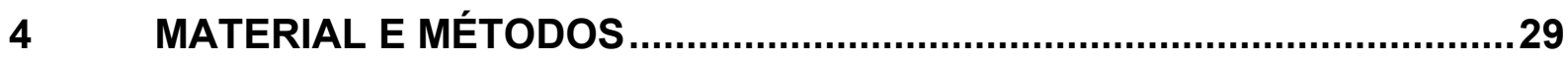

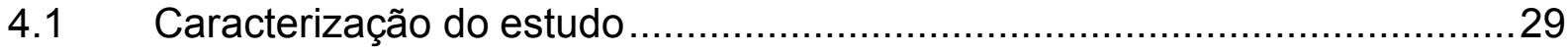

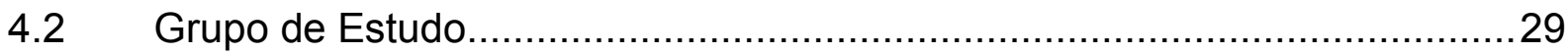

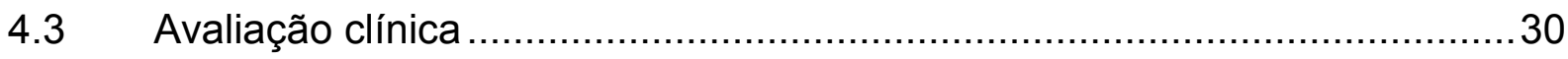

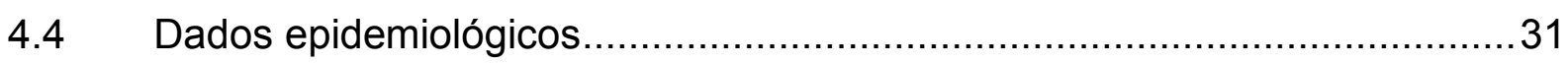

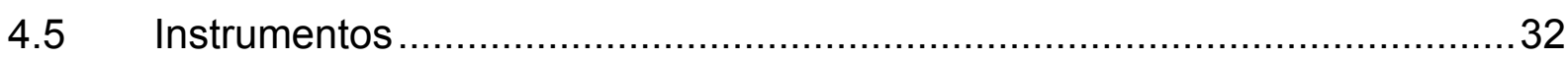

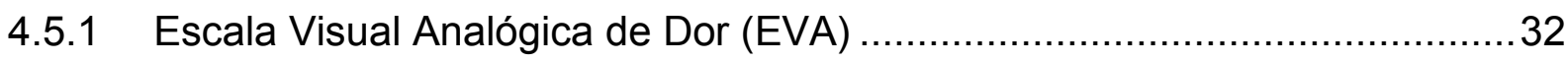

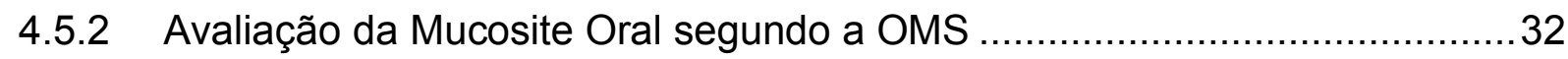

4.5.3 Avaliação da Mucosite Oral segundo a Oral Mucositis Assessment Scale

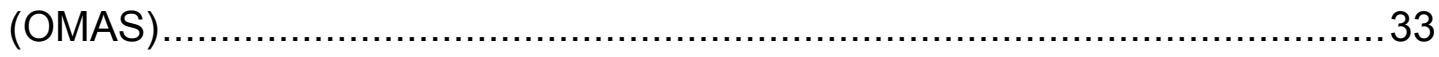

4.5.4 Avaliação do limiar de dor à pressão - Algômetro Analógico.........................33

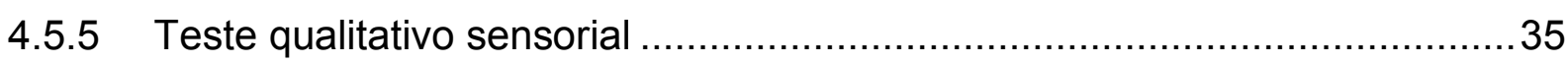

4.5.6 Depression, Anxiety and Stress Scale-21 (DASS-21) ……........................ 35

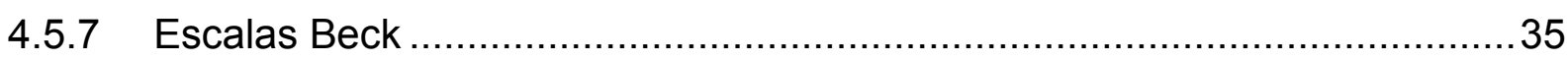

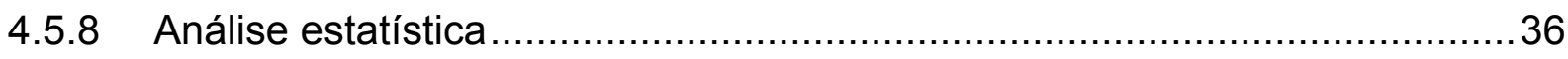





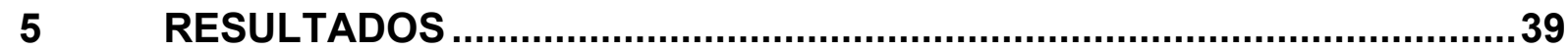

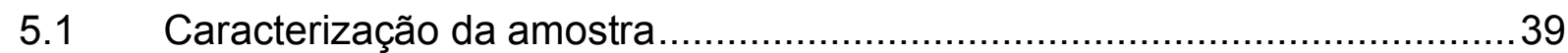

5.2 Depression, Anxiety and Stress Scale-21 (DASS-21) ...........................40

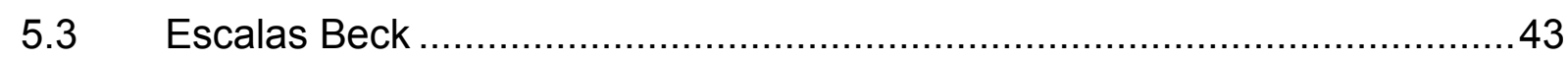

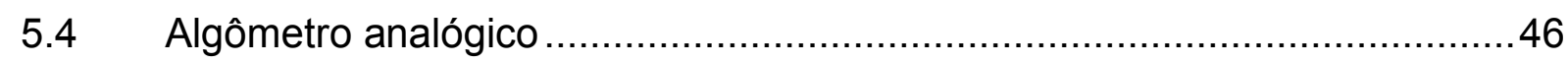

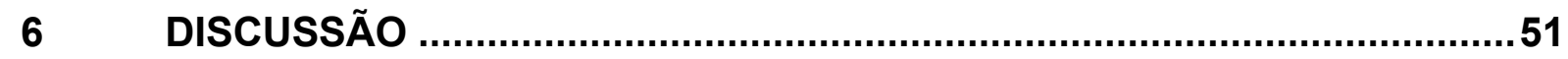

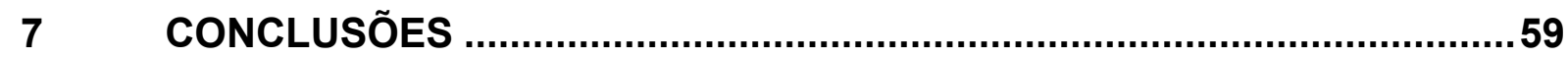

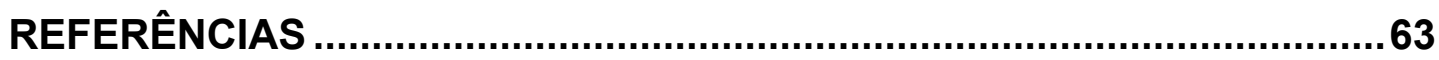

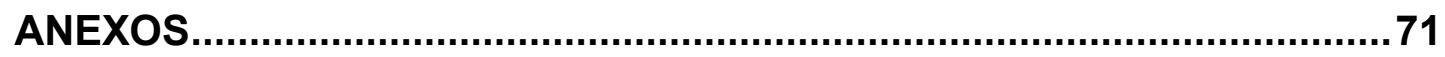



1 INTRODUÇÃO 



\section{INTRODUÇÃO}

Dentre os mais variados efeitos colaterais que resultam da quimioterapia, a complicação que aparece com mais frequência é a mucosite oral (MO). Alguns artigos recentes trazem informações sobre a MO no intuito de não apenas identificar esse acometimento, mas para estudar e propor tratamentos que possam retardar o surgimento dessas lesões ou ainda proporcionar um efeito antiinflamatório como ocorre com o uso da laserterapia (SANTOS, DIAS, BARROS, FREITAS, 2010. PINHEIRO, TOLENTINO, 2018. VELOSO, GUEDES, 2019). A MO é a inflamação da parte interna da boca, resultando em dor, no surgimento de várias feridas e de difícil cicatrização. A MO afeta diretamente a qualidade de vida do indivíduo, pois além de ter várias feridas, também pode ocorrer a perda do paladar (ageusia), ou ainda sentir o sabor como desagradável (disgeusia), a dificuldade da fala (SANTOS et al., 2009; PINHO et al., 2010).

Já em relação ao estado emocional, sabe-se sobre o risco aumentado para o desenvolvimento de depressão em indivíduos com câncer, quando comparados com a população geral (AVELAR et al., 2006; BOTTINO, FRÁGUAS, GATTAZ, 2009).

Para esta pesquisa, buscou-se em bases de dados científicas estudos que abordassem a relação do tratamento quimioterápico, $\mathrm{MO}$, depressão, ansiedade e diminuição do limiar de dor, entretanto, foi encontrado na literatura apenas um artigo que abordou a relação entre mucosite oral, depressão e ansiedade, sendo ele: "Assessment of Anxiety and Drepression in Oral Mucositis Patients Undergoing Cancer Chemoradiotherapy: A Randomized Cross-sectional Study" (CHAITANYA et al, 2016).

Ao verificar essa escassez na literatura, observou-se a necessidade de realizar um estudo que mostrasse como está o paciente oncológico antes de receber a quimioterapia e após fazer seu uso identificar os efeitos colaterais que advêm do tratamento antineoplásico, principalmente abordando os aspectos psicológicos.

Por entender que há prejuízos não somente físico, mas também psicológico em pacientes com câncer, procurou-se identificar quais aspectos psicológicos estão presentes em pacientes oncológicos acometidos por MO e dor. 

7 CONCLUSÕES 



\section{CONCLUSÕES}

Este estudo possibilitou identificar a presença da depressão e ansiedade em pacientes oncológicos, com mucosite oral, após o uso de quimioterápicos. Foi mensurado o limiar de dor dos pacientes por meio de alguns testes, que não obtiveram relevância estatística, entretanto, quando comparados separadamente, observou-se aumento do limiar de dor após o uso de quimioterápicos; observou-se também que a sensação subjetiva (EVA) de dor é diferente quando comparada com a sensação objetiva (algômetro analógico) da dor. Não houve relevância estatística ao comparar os aspectos psicológicos com o limiar de dor. 



\section{REFERÊNCIAS}





\section{REFERÊNCIAS}

ALVES, F. A. Plano de Tratamento Odontológico em Pacientes com Neoplasias Malignas de Cabeça e Pescoço. In: SANTOS, P. S. S. SOARES JUNIOR, L. A. V. Medicina Bucal: A Prática na Odontologia Hospitalar. São Paulo. Santos Editora. 2012.AVELAR, A. M. B., et al. Qualidade de vida, ansiedade e depressão em mulheres com câncer de mama antes e após a cirurgia. Rev. Ciênc. Méd., v. 15, n. 1, p. 11-20, 2006. Disponível em: http://seer.sis.puccampinas.edu.br/seer/index.php/cienciasmedicas/article/viewFile/1131/1106. Acesso em 19 de Fev de 2020.

ALVES, R. F, et al. A saúde do homem na interface com a Psicologia da Saúde. In: ALVES, R. F. Psicologia da Saúde: teoria, intervenção e pesquisa. Campina Grande. EDUEPB. 346 p. 2011.

BOTTINO, S. M. B. FRÁGUAS, R. GATTAZ, W. F. Depressão e câncer. Rev. Psiq. Clín., v. 36 (supl. 3), p. 109-115, 2009. Disponível em:

$<$ http://www.scielo.br/scielo.php?pid=S0101-

60832009000900007\&script=sci_arttext>. Acesso em 19 de Fev de 2020.

BRASIL. Instituto Nacional de Câncer José Alencar Gomes da Silva. Controle do Câncer: uma proposta de integração ensino-serviço. Disponível em: <http://www.inca.gov.br/conteudo_view.asp?id=101>. Rio de Janeiro: INCA. 1993. Acesso em 2 de Nov de 2019.

BRASIL. Instituto Nacional de Câncer José Alencar Gomes da Silva. O que é câncer? Disponível em: <https://www.inca.gov.br/o-que-e-cancer>. Rio de Janeiro: INCA. 2019. Acesso em 5 de Fev de 2020.

BRASIL. Instituto Nacional de Câncer José Alencar Gomes da Silva. Estimativa 2020: incidência de câncer no Brasil. Rio de Janeiro: INCA, 2019. Disponível em: <https://www.inca.gov.br/sites/ufu.sti.inca.local/files//media/document//estimativa2020-incidencia-de-cancer-no-brasil.pdf>. Acesso em 5 de Fev de 2020.

BRASIL. Instituto Nacional do Câncer José Alencar Gomes da Silva (INCA).

Estimativa 2018: incidência de câncer no Brasil. Rio de Janeiro: INCA, 2017. Disponível em:

https://www.inca.gov.br/sites/ufu.sti.inca.local/files//media/document//estimativaincidencia-de-cancer-no-brasil-2018.pdf. Acesso em 2 de Nov de 2019. 
BRASIL. Ministério da Saúde. Câncer: sintomas, causas, tipos e tratamentos. Disponível em: <https://www.saude.gov.br/saude-de-a-z/cancer>. 2020. Acesso em 5 de Fev de 2020.

CARVALHO, M. M. M. J. Psico-Oncologia: História, Características e Desafios. Psicologia USP. v. 13, n. 1, p. 151-166. 2002. Disponível em: < http://www.scielo.br/scielo.php?script=sci_arttext\&pid=S0103-65642002000100008>. Acesso em 13 de Dez de 209.

CFP, Conselho Federal de Psicologia. Teste das Fábulas tem parecer desfavorável e não pode mais ser utilizado. Psicólogo deve consultar regularmente o Satepsi para se atualizar sobre os testes válidos, s/d. Disponível em:

http://www.crpsp.org.br/portal/comunicacao/jornal_crp/167/frames/fr_orientacao2.asp x. Acesso em 13 de fev de 2020.

CURRA, M. SOARES JUNIOR, L. A. V. MARTINS, M. D. SANTOS, P. S. S. Protocolos quimioterápicos e incidência de mucosite oral. Revisão integrativa. Einstein, v. 16, n. 1, p. 1-9, 2018. Disponível em: < https://journal.einstein.br/wpcontent/uploads/articles_xml/1679-4508-eins-16-01-eRW4007/1679-4508-eins-1601-eRW4007-pt.x54418.pdf>. Acesso em 07 de fev de 2020.

DELGALARRONDO, P. Síndromes depressivas. In: DELGALARRONDO, P. Psicopatologia e semiologia dos transtornos mentais. $2^{\circ}$ edição. Porto Alegre: Artimed, 2008

DUARTE, B. G. MACIEL, A. P. GONÇALVES, E. S. SANTOS, P. S. S. Avaliação perioperatória de indivíduos em quimioterapia com necessidade de intervenção cirúrgica odontológica. Arquivos Médicos, v. 63, n. 2, p. 105-109. São Paulo. 2018. Disponível em: https://doi.org/10.26432/1809-3019.2018.63.2.105. Acesso em 06 de Jan de 2020.

EDUARDO, F. P. et al. Influência dos cuidados odontológicos acompanhados de laserterapia sobre a mucosite oral durante transplante alogênico de células hematopoiéticas: estudo retrospectivo. Einstein. São Paulo, v.9, n.2, pp.201206. 2011. Disponível em:

<http://www.scielo.br/scielo.php?pid=S167945082011000200201\&script=sci_abstrac t\&tlng=pt>. Acesso em 20 de Jan de 2019.

EDUARDO, F. P. BENZINELLI, L. M. CORREAA, L. Terapia laser de baixa potência na mucosite oral. In: GARCEZ, A. S. RIBEIRO, M. S. NÚÑEZ, S. C. Laser de baixa potência: princípios básicos e aplicações clínicas na odontologia. Rio de Janeiro: Saunders Elsevier. 2012. 
EILERS, J. EPSTEIN, J. B. Assessment and measurement of oral mucositis.

Seminars in Oncology Nursing, v. 20, n. 1. 2004. Disponível em:

$<$ https://ac.elscdn.com/S0749208103001360/1-s2.0-

S0749208103001360main.pdf?_tid=18db50f3-e777-4c12-

8c326f94af4c6150\&acdnat=1524161092_25f29e052fb5fa198570bb259e58b541>.

Acesso em 19 de Jan de 2020.

ERDOĞAN, E. DEMIR, S. ÇALIŞKAN, B. B. BAYRAK, N. G. Effect of psychological care given to the women who underwent hysterectomy before and after the surgery on depressive symptoms, anxiety and the body image levels. J Obstet Gynaecol, 2019. doi: 10.1080/01443615.2019.1678574. Acesso em 28 de Fev de 2020.

GANDINI, R. C. MARTINS, M. C. F. RIBEIRO, M. P. SANTOS, D. T. G. Inventário de depressão de Beck - BDI: validação fatorial para mulheres com câncer. Psico-USF, v. 12, n. 1, 2007. Disponível em:

<http://www.scielo.br/pdf/pusf/v12n1/v12n1a04.pdf>. Acesso em 27 de Fev de 2020.

GIMENES, M. G. G. Definição, foco de estudo e intervenção. In: CARVALHO, M. M. M. J. Introdução à psiconcologia. Livro Pleno. 2003.

HESPANHOL, F. L. et al. Manifestações bucais em pacientes submetidos à quimioterapia. Ciência e Saúde Coletiva, v. 15, n. 1, p. 1085-1094. 2010. Disponível em:

<http://www.scielo.br/scielo.php?script=sci_arttext\&pid=S141381232010000700016\& Ing=pt\&nrm=iso>. Acesso em 31 Jan 2020.

INSTITUTO ONCOGUIA. Ansiedade. Disponível em: <http://www.oncoguia.org.br/conteudo/ansiedade/1322/109/>. Acesso em 7 de Fev de 2020.

KELNER, N. CASTRO, J. F. L. Laser de baixa intensidade no tratamento da mucosite oral induzida pela radioterapia: relato de casos clínicos. Revista Brasileira de Cancerologia, v. 53, n. 1, p. 29-33. 2007. Disponível em:

<http://www.inca.gov.br/rbc/n_53/v01/pdf/relato_caso1.pdf>. Acesso em 13 de Fev de 2020.

LOVIBOND, P. F. LOVIBOND, S. H. The structure of negative emotional states: comparison of the Depression Anxiety Stress Scales (DASS) with the Beck Depression and Anxiety Inventories. Behav. Res. Ther., v. 33, n. 3, p. 335-343. Acesso em 15 de Jan de 2020.

MANUAL DIAGNÓSTICO E ESTATÍSTICO DE TRANSTORNOS MENTAIS: DSM-V. American Psychiatric Association. $5^{\circ}$ edição. Porto Alegre: Artmed, 2014. 
McMULLEN, M. LAU, P. K. H. TAYLOR, S. McTIGUE, J. COZINHEIRO, A. BAMBLETT, M. HASANI, A. JOHNSON, C. E. Factors associated with psychological distress amongst outpatient chemotherapy patients: An analysis of depression, anxiety and stress using the DASS-21. Appl Nurs Res, p. 45-50, 2018. Disponível em: <https://www.ncbi.nlm.nih.gov/pubmed/29579498>. Acesso em 27 de Fev de 2020.

NAIDU, M. U et al. Chemotherapy-induced and/or radiation therapy induced oral mucositis-complicating the treatment of cancer. Neoplasia, v. 6, n. 5, p. 423-431. 2004. Disponível em: <https://www.ncbi.nlm.nih.gov/pubmed/15548350>. Acesso em 23 de Nov de 2019.

NAZARÉ, M. S. L. SILVA, J. A. M. G. NAVEGA, M. T. FAGNELLO-NAVEGA, F. R. Comparision of pain threshold and duration of pain perception in men and women of diferente ages. Fisioter. Mov, v. 27, n. 1, p. 77-84, 2014. Disponível em: http://www.scielo.br/pdf/fm/v27n1/0103-5150-fm-27-01-0077.pdf. Acesso em 13 de Fev de 2020.

ORGANIZAÇÃO PAN-AMERICANA DA SAÚDE (PAHO). Campanha: "Vamos conversar". 2017. Disponível em:

http://www.paho.org/bra/index.php?option=com_content\&view=article\&id=5385:comd epressao-no-topo-da-lista-de-causas-de-problemas-de-saude-oms-lancaacampanha-vamos-conversar\&Itemid=839. Acesso em 26 de Nov de 2019.

PINHEIRO, I. H. S. TOLENTINO, E. S. Mucosite oral induzida por quimioterapia. Revista FAIPE, v. 8, n. 2, p. 30-42, 2018. Disponível em: http://revistafaipe.lifesistemas.com.br/index.php/RFAIPE/article/view/100/96. Acesso em 18 de Fev de 2020.

PINHO, A. P, et al. Mucosite no Paciente em Tratamento de Câncer. Science in Health. v. 1, n. 3, p. 145-160. 2010. Disponível em:

$<$ http://arquivos.cruzeirodosuleducacional.edu.br/principal/new/revista_scienceinhealt h/03_set_dez_2010/science_145_60.pdf>. Acesso em 31 Nov 2019.

SANTOS, P. S. S. DIAS, R. R. BARROS, J. C. A. FREITAS, R. R. Prevenção da mucosite oral utilizando laser terapêutico. Arq Med Hosp Fac Cienc Med Santa Casa São Paulo, v. 55, n. 1, p. 7-11, 2010. Disponível em: < https://www.researchgate.net/publication/234111285_Prevencao_da_mucosite_oral_ utilizando_LASER_terapeutico_Prevention_of_oral_mucositis_using_LASER_therap y>. Acesso em 7 de Fev de $20 \overline{2} 0$.

SANTOS, P. S. S. MESSAGGI, A C. MANTESSO, A. MAGALHÃES, M. H. C. G. Mucosite oral: perspectivas atuais na prevenção e tratamento. Revista Gaúcha de Odontologia. v. 57, n. 3, p. 339-344. 2009. Disponível em: <http://bases.bireme.br/cgibin/wxislind.exe/iah/online/?IsisScript=iah/iah.xis\&src=goo 
gle\&base $=$ LILACS\&lang=p \&nextAction=Ink\&exprSearch=527917\&indexSearch=ID> . Acesso em 2 de Fev de 2020.

SARDÁ JÚNIOR, J. Dor: visão biopsicossocial. In: PORTNOI, A. G. A psicologia da dor. $1^{\circ}$ ed. São Paulo: Guanabara Koogan, 2014.

SATIN, J. R., LINDEN, W. PHILLIPS, M. J. Depression as a predictor of disease progression and mortality in câncer patients: A meta-analysis. Câncer. V. 111, n. 22, p. 5349-5361. 2009. Disponível em:

<https://www.ncbi.nlm.nih.gov/pubmed/19753617>. Acesso em 12 de Dez de 2019.

SCANNAVIO, C. S. S, et al. A atuação do psicólogo no hospital de câncer de Barretos. Psicologia USP. v. 24, n. 1, p. 35-53. 2013. Disponível em: < http://www.redalyc.org/html/3051/305128931003/>. Acesso em 29 de Nov de 2019.

SCORSOLINI-COMIN, et al. Tornar-se psicólogo: experiência de estágio de PsicoOncologia em equipe multiprofissional de saúde. Revista Brasileira de Orientação Profissional, v. 9, n. 2, p. 113-125. 2008. Disponível em: < http://pepsic.bvsalud.org/scielo.php?script=sci_arttext\&pid=S1679339020080002000 10>. Acesso em 12 de Dez de 2019.

SILVA, T. D. FOGER, D. GARDINAL-PIZATO, E. C. SANTOS, P. S. S. Transmissão do diagnóstico de câncer sob a perspectiva da observação psicológica. Arch. Health Invest., v. 8, n. 8, p. 448-450, 2019. Acesso em:

$<$ http://www.archhealthinvestigation.com.br/ArcHI/article/view/4668/pdf>. Acesso em 9 de Dez de 2019.

SIQUEIRA, K. M. BARBOSA, M. A. BOEMER, M. R. O vivenciar a situação de ser com câncer: alguns des-velamentos. Rev Latino-am Enfermagem, v. 15, n. 4, 2007. Acesso em: <http://www.scielo.br/scielo.php?pid=S0104-

11692007000400013\&script=sci_arttext\&tlng=pt>. Acesso em 9 de Fev de 2020.

SOUZA, B. F. et al. Pacientes em uso de quimioterápicos: depressão e adesão ao tratamento. Rev Esc Enferm USP, v. 47, n. 1, p. 61-68, 2013. Disponível em: <http://www.periodicos.usp.br/reeusp/article/view/52853/56758>. Acesso em 9 de Fev de 2020.

TROTTI, A. et al. Mucositis incidence, severity and associated outcomes in patients with head and neck cancer receiving radiotherapy with or without chemotherapy: a systematic literature review. Radiother Oncol. v. 66, n. 3, p. 253-262. 2003. Disponível em: < https://www.ncbi.nlm.nih.gov/pubmed/12742264>. Acesso em 14 de Set de 2019. 
VANCONCELOS, A. S. COSTA, C. BARBOSA, L. N. F. Do transtorno de ansiedade ao câncer. Rev SBPH, v. 11, n. 2, p. 51-71, 2008. Disponível em: < http://pepsic.bvsalud.org/scielo.php?script=sci_arttext\&pid=S151608582008000200006>. Acesso em 7 de Fev de 2020.

VEIT, M. T. CARVALHO, V. A. Psico-Oncologia: definições e área de atuação. In: CARVALHO, V. A. (org). Temas em psico-oncologia. São Paulo. Summus Editorial. 2008

WORLD HALTH ORGANIZATION. Who handbook for reporting results of cancer treatment. 1979. Disponível em:

<http://apps.who.int/iris/bitstream/handle/10665/37200/WHO_OFFSET_48.pdf?sequ ence=1\&isAllowed=y>. Acesso em 13 de Set de 2019.

ZAMAGNANI, D. R. BANACO, R. A. Um panorama analítico-comportamental sobre os transtornos de ansiedade. Rev. bras. ter. comport. cogn, v. 7, n. 1, p. 77-92. 2005. Disponível em:

http://pepsic.bvsalud.org/scielo.php?script=sci_arttext\&pid=S1517-

55452005000100009. Acesso em 9 de Fev de 2020. 
AnEXos 



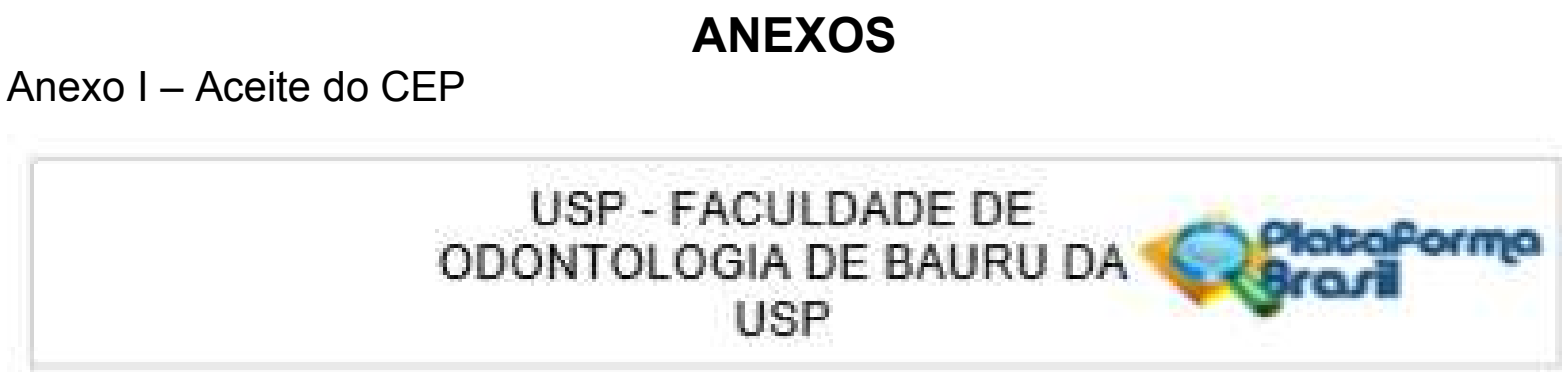

\section{PARECER CONSUBSTANCIADO DO CEP}

\section{DADOS DO PROJETO DE PESQUISA}

Titulo da Pesquisa: Aspectos psicolópicos, qualidade de vida e limiar de dor em individuos com cáncer com risco de desenvolver muocsite oral quimicinduzida

Pesquiaador: PALLO SERGIO DA SLVA SANTOS

Area Tematica:

Versao: 1

CAAE: 00153D18.6.0000:5417

Instituiça Proponante: Universidade de Sao Paulo

Patrocinador Principat: Financiamento Prógrio

\section{DADOS DO PARECER}

Numero do Parecer: 2.976 .899

\section{ApresentaçBo do Projeto:}

Pacienles oncológicos submelidos Aa quitriolerapia podem desenvolver mucosile oral, dependendo da quantidade e foxicidade das terapas utlilzadas. A mucosile orai e a inflamecao da parte intema mucosa. feaultando no surgiments de eritemas eiou ullceras de dilicil cicabrizaça. A lieratura aponta a relaçás entre câncer 6 depreas 60 , havendo assim, maior predisposiça do individuo com a necplasia: a depressbo $e$ caracterizada por desânima e humor triste, sendo as sintamas mais frequentes o humor deprimido, pessimisma, autoestima rebaikada, aimento ou diminuiça do apethe, heónis ou aumento do sono, entre cutros. Para tanto, husca-se proporciarar qualidade de vida de forma multidimensional, atrangendo o ser biopsicossocial-espirifual. Esse frabatho term como objetivo identificar falores psicoldgicos, tais como ansiedach, depressbo e nstresse am pecientes cncoićgicos acometidos por mucosle cral. Trata-se ds uma

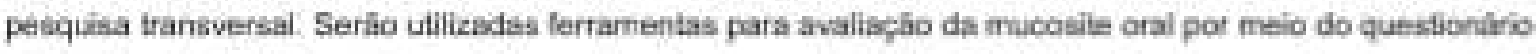
adotado pela OMS e pelo Oral Mucosilis Assessment Scale (OMAS) e da depressaso por meio do Depresssion, Anxiety and Stress Scale.21 (DASS-21), Escaias Beck: Short Form Heaith Survey (SF.36); Escala Visual Analógica; OHIP-14; Avalisçso do limiar de dor à pressas; e o teste qualitativo serisotial.

\section{Objettivo da Pesquisa:}

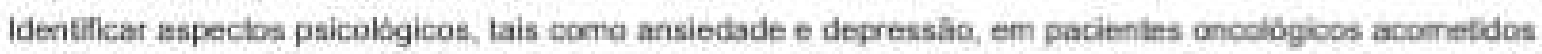
por mucosibe orat.

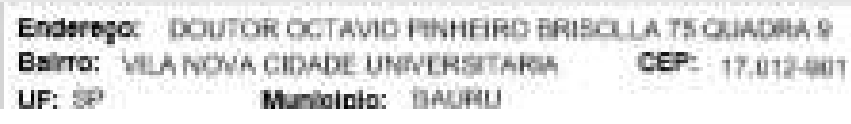


Anexo II - Ficha utilizada

\section{$1^{\circ}$ MOMENTO}

Nome:

Data de nascimento:

$\mathrm{N}^{\circ}$ prontuário:

Local de nascimento:

Data:

Endereço:

Cidade:

Escolaridade:

Profissão:

Religião:

Condição conjugal:

Doença de base:

Comorbidades:

1- EVA de dor:

2- Avaliação OMS:

3- Avaliação OMAS:

4- Algômetro:

\begin{tabular}{|l|l|l|l|l|}
\hline VALOR 1 & VALOR 2 & VALOR 3 & SOMATÓRIA & MÉDIA \\
\hline & & & & \\
\hline
\end{tabular}

5- Teste qualitativo sensorial:

\begin{tabular}{|l|l|l|l|}
\hline Mucosa jugal D & Normosensibilidade & Hipersensibilidade & Hiposensibilidade \\
\hline Cotonete & & & \\
\hline Espátula & & & \\
\hline Palito & & & \\
\hline
\end{tabular}




\begin{tabular}{|l|l|l|l|}
\hline Mucosa jugal E & Normosensibilidade & Hipersensibilidade & Hiposensibilidade \\
\hline Cotonete & & & \\
\hline Espátula & & & \\
\hline Palito & & & \\
\hline
\end{tabular}

6- DASS-21:

DeprDepressão:

Ansiedade:

Estresse:

7- Escalas Beck:

BDI:

BAI:

BSI:

BHS:

\section{$2^{\circ}$ MOMENTO}

Nome:

$\mathrm{N}^{\circ}$ prontuário:

Data:

1- EVA de dor:

2- Avaliação OMS:

3- Avaliação OMAS:

4- Algômetro:

\begin{tabular}{|l|l|l|l|l|}
\hline VALOR 1 & VALOR 2 & VALOR 3 & SOMATÓRIA & MÉDIA \\
\hline & & & & \\
\hline
\end{tabular}


5- Teste qualitativo sensorial:

\begin{tabular}{|l|l|l|l|}
\hline Mucosa jugal D & Normosensibilidade & Hipersensibilidade & Hiposensibilidade \\
\hline Cotonete & & & \\
\hline Espátula & & & \\
\hline Palito & & & \\
\hline Mucosa jugal E & Normosensibilidade & Hipersensibilidade & Hiposensibilidade \\
\hline Cotonete & & & \\
\hline Espátula & & & \\
\hline Palito & & & \\
\hline
\end{tabular}

6- DASS-21:

Depressão:

Ansiedade:

Estresse:

7- Escalas Beck:

BDI:

BAI:

BSI:

BHS:

$3^{\circ}$ MOMENTO

Nome:

$\mathrm{N}^{\circ}$ prontuário:

Data:

1- EVA de dor:

2- Avaliação OMS: 
3- DASS-21:

Depressão:

Ansiedade:

Estresse: 
Anexo III - DASS-21

NOME:

DATA:

$\mathrm{N}^{\circ}$ de registro:

DASS - 21 Versão traduzida e validada para o português do Brasil Autores: Vignola, R.C.B. \& Tucci, A.M.

\section{Instruções}

Por favor, leia cuidadosamente cada uma das afirmações abaixo e circule onúmero apropriado $0,1,2$ ou 3 que indique o quanto ela se aplicou a você durante a última semana, conforma a indicação a seguir:

0 Não se aplicou de maneira alguma

1 Aplicou-se em algum grau ou por pouco tempo

2 Aplicou-se em um grau considerável ou por uma boa parte do tempo

3 Aplicou-se muito ou na maioria do tempo

D A $\quad E$

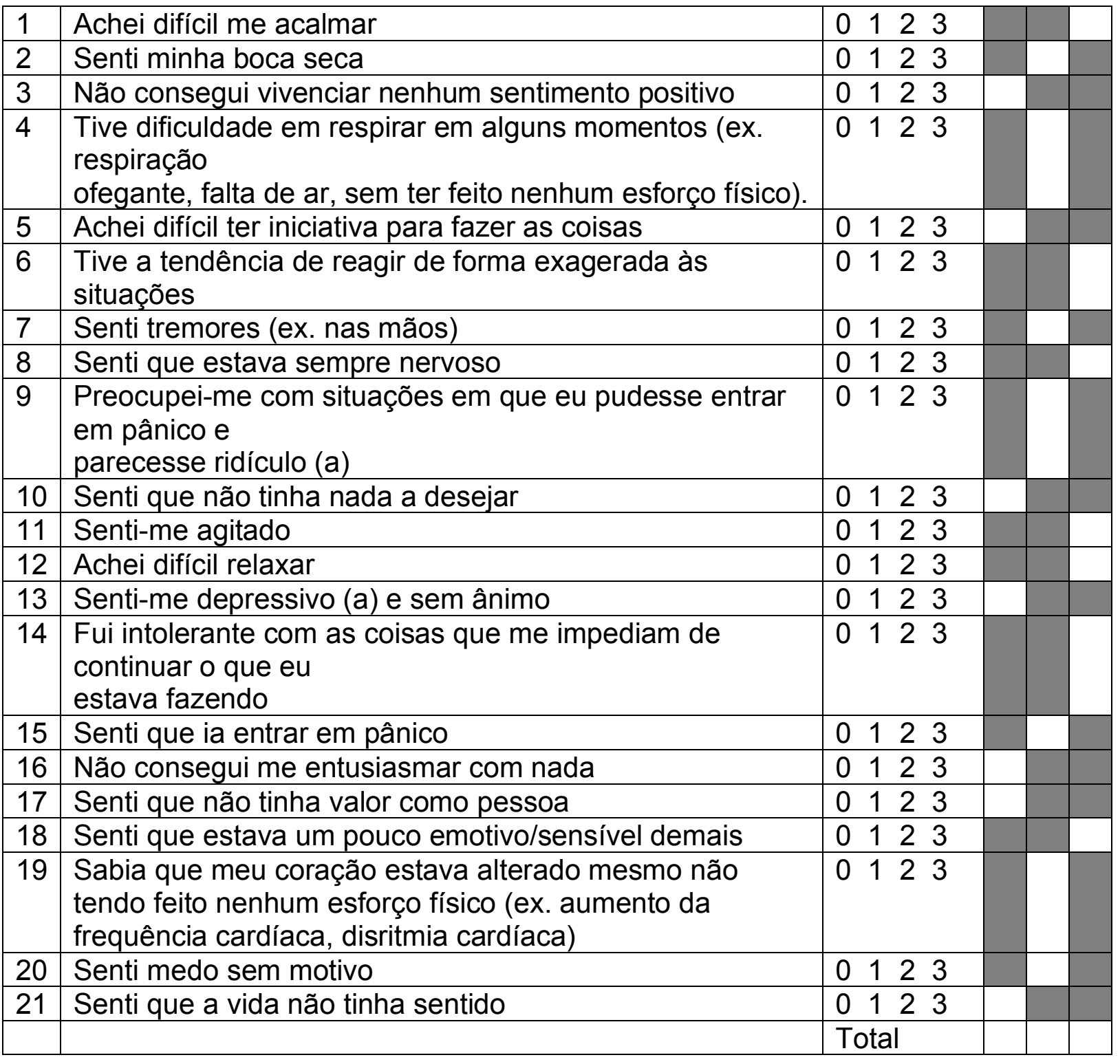


DASS - 21 SCORE

Estresse

Score
Depressão

Score
Ansiedade

Score

\begin{tabular}{|l|l|l|l|}
\hline & Depressão & Ansiedade & Estresse \\
\hline Normal & $0-4$ & $0-3$ & $0-7$ \\
\hline Leve & $5-6$ & $4-5$ & $8-9$ \\
\hline Moderado & $7-10$ & $6-7$ & $10-12$ \\
\hline Grave & $11-13$ & $8-9$ & $13-16$ \\
\hline Severo & $14+$ & $10+$ & $17+$ \\
\hline
\end{tabular}




\section{Escalas Beck:}

\section{Anexo IV - BDI - Beck Depression Inventory}

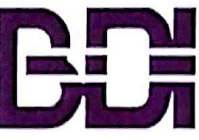

Data:

Nome:

Estado Civil:

Idade:

Sexo:

Ocupação:

Escolaridade:

Este questionário consiste em 21 grupos de afirmações. Depois de ler cuidadosamente cada grupo, faça um círculo em torno do número $(0,1,2$ ou 3$)$ próximo à afirmação, em cada grupo, que descreve melhor a maneira que você tem se sentido na última semana, incluindo hoje. Se várias afirmações num grupo parecerem se aplicar igualmente bem, faça um círculo em cada uma. Tome o cuidado de ler todas as afirmações, em cada grupo, antes de fazer a sua escolha.

10 Não me sinto triste.

1 Eu me sinto triste.

2 Estou sempre triste e não consigo sair disto.

3 Estou tão triste ou infeliz que não consigo suportar.

2 Não estou especialmente desanimado quanto ao futuro.

1 Eu me sinto desanimado quanto ao futuro.

2 Acho que nada tenho a esperar.

3 Acho o futuro sem esperança e tenho a impressão de que as coisas não podem melhorar.

3 Não me sinto um fracasso.

1 Acho que fracassei mais do que uma pessoa comum.

2 Quando olho para trás, na minha vida, tudo o que posso ver é um monte de fracassos.

3 Acho que, como pessoa, sou um completo fracasso.

40 Tenho tanto prazer em tudo como antes.

1 Não sinto mais prazer nas coisas como antes.

2 Não encontro um prazer real em mais nada.

3 Estou insatisfeito ou aborrecido com tudo.

50 Não me sinto especialmente culpado.

1 Eu me sinto culpado grande parte do tempo.

2 Eu me sinto culpado na maior parte do tempo.

3 Eu me sinto sempre culpado.

6 Não acho que esteja sendo punido.

1 Acho que posso ser punido.

2 Creio que vou ser punido.

3 Acho que estou sendo punido.

70 Não me sinto decepcionado comigo mesmo.

1 Estou decepcionado comigo mesmo.

2 Estou enojado de mim.

3 Eu me odeio.
8 - Não me sinto de qualquer modo pior que os outros

1 Sou crítico em relação a mim por minhas fraquezas ou erros.

2 Eu me culpo sempre por minhas falhas.

3 Eu me culpo por tudo de mal que acontece.

90 Não tenho quaisquer idéias de me matar.

1 Tenho idéias de me matar, mas não as executaria.

2 Gostaria de me matar.

3 Eu me mataria se tivesse oportunidade.

100 Não choro mais que o habitual.

1 Choro mais agora do que costumava.

2 Agora, choro o tempo todo.

3 Costumava ser capaz de chorar, mas agora não consigo, mesmo que o queira.

11 Não sou mais irritado agora do que já fui.

I Fico aborrecido ou irritado mais facilmente do que costumava.

2 Agora, eu me sinto irritado o tempo todo.

3 Não me irrito mais com coisas que costumavam me irritar.

12 Não perdi o interesse pelas outras pessoas.

1 Estou menos interessado pelas outras pessoas do que costumava estar.

2 Perdi a maior parte do meu interesse pelas outra pessoas.

3 Perdi todo o interesse pelas outras pessoas.

13 ) Tomo decisões tão bem quanto antes.

I Adio as tomadas de decisões mais do que costumava.

2 Tenho mais dificuldades de tomar decisões do que antes.

3 Absolutamente não consigo mais tomar decisões. 


\section{Continuação do BDI}

14 ) Não acho que de qualquer modo pareço pior do que antes.

1 Estou preocupado em estar parecendo velho ou sem atrativo.

2 Acho que há mudanças permanentes na minha aparência, que me fazem parecer sem atrativo.

3 Acredito que pareço feio.

15 Posso trabalhar tão bem quanto antes.

1 É preciso algum esforço extra para fazer alguma coisa.

2 Tenho que me esforçar muito para fazer alguma coisa.

3 Não consigo mais fazer qualquer trabalho.

16 Consigo dormir tão bem como o habitual

Não durmo tão bem como costumava.

2 Acordo 1 a 2 horas mais cedo do que habitualmente e acho difícil voltar a dormir.

3 Acordo várias horas mais cedo do que costumava e não consigo voltar a dormir.

17 0 Não fico mais cansado do que o habitual.

1 Fico cansado mais facilmente do que costumava.

2 Fico cansado em fazer qualquer coisa.

3 Estou cansado demais para fazer qualquer coisa.

18 O meu apetite não está pior do que o habitual.

1 Meu apetite não é tão bom como costumava ser.

Meu apetite é muito pior agora.

3 Absolutamente não tenho mais apetite.
19 Não tenho perdido muito peso se é que perdi algum recentemente.

1 Perdi mais do que 2 quilos e meio.

2 Perdi mais do que 5 quilos.

3 Perdi mais do que 7 quilos.

Estou tentando perder peso de propósito, comendo menos: Sim Não

20 Não estou mais preocupado com a minha saúde do que o habitual.

1 Estou preocupado com problemas físicos, tais como dores, indisposição do estômago ou constipação.

2 Estou muito preocupado com problemas físicos $\mathrm{e}$ é difícil pensar em outra coisa.

3 Estou tão preocupado com meus problemas físicos que não consigo pensar em qualquer outra coisa.

21 Não notei qualquer mudança recente no meu interesse por sexo.

1 Estou menos interessado por sexo do que costumava.

2 Estou muito menos interessado por sexo agora.

3 Perdi completamente o interesse por sexo.

Subtotal da Página 2

Subtotal da Página 1

Escore Total. 
Anexo V - BAI - Beck Anxiety Inventory

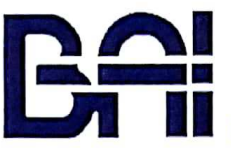

Data:

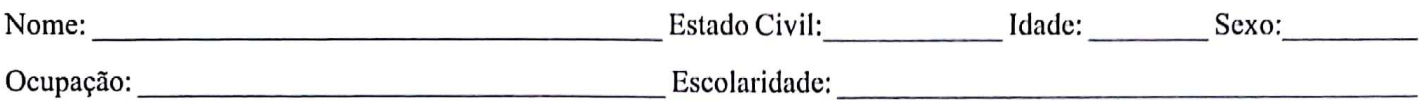

Abaixo está uma lista de sintomas comuns de ansiedade. Por favor, leia cuidadosamente cada item da lista. Identifique o quanto você tem sido incomodado por cada sintoma durante a última semana, incluindo hoje, colocando um " $x$ " no espaço correspondente, na mesma linha de cada sintoma.

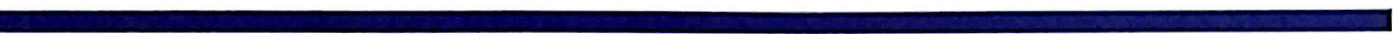

1. Dormência ou formigamento.

2. Sensação de calor.

3. Tremores nas pernas.

4. Incapaz de relaxar.

5. Medo que aconteça o pior.

6. Atordoado ou tonto.

7. Palpitação ou aceleração do coração.

8. Sem equilibrio.

9. Aterrorizado.

10. Nervoso.

11. Sensação de sufocação.

12. Tremores nas mãos.

13. Trêmulo.

14. Medo de perder o controle.

15. Dificuldade de respirar.

16. Medo de morrer.

17. Assustado.

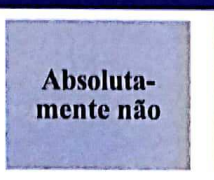

\begin{tabular}{|l|c|}
\hline $\begin{array}{c}\text { Levemente } \\
\text { Não me inco- } \\
\text { modou muito }\end{array}$ & $\begin{array}{c}\text { Moderada- } \\
\text { mente } \\
\text { Foi muito de- } \\
\text { sagradável mas } \\
\text { pude suportar }\end{array}$ \\
\hline
\end{tabular}

Gravemente

Dificilmente pude suportar

pude suportar
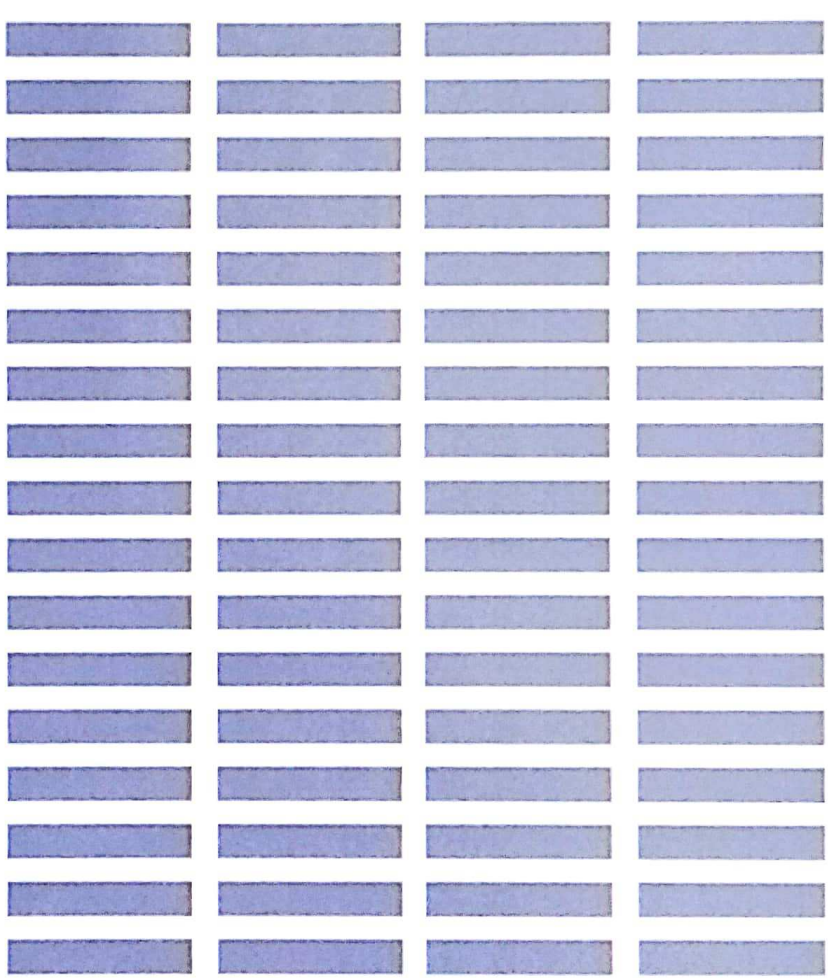

18. Indigestão ou desconforto no abdômen.

19. Sensação de desmaio.

20. Rosto afogueado.

21. Suor (não devido ao calor).
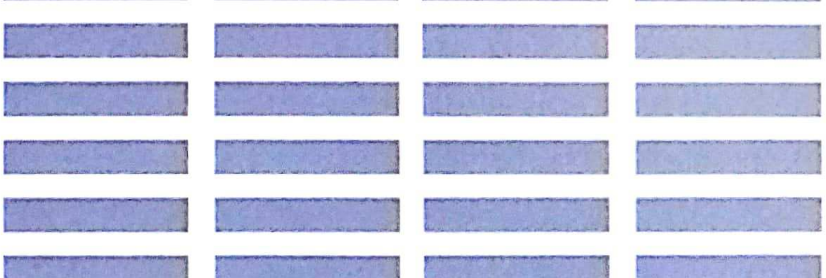

Total

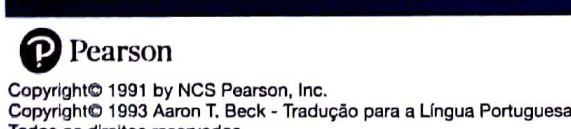

Copyrighto 1991 by NCS Pearson, Inc. Todos os direitos reservados. 


\section{Anexo VI - BHS - Beck Hopelessness Scale}

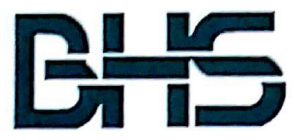

Data:

Nome:

Estado Civil:

Idade:

Sexo:

Ocupação:

Escolaridade:

Este questionário consiste em 20 afirmações. Por favor, leía as afirmações cuidadosamente, uma por uma, Se a afirmação descreve a sua atitude na áltima semana, incluindo hoje, escureça o círculo com "C", indicando CERTO, na mesma linha da afirmação. Se a afirmação não descreve a sua atitude, escureça o círculo com "E", indicando ERRADO, na mesma linha da afirmação. Por favor, procure ler cuidadosamente cada afirmação.

1. Penso no futuro com esperança e entusiasmo.

(C) (E)

2. Seria melhor desistir, porque nada há que eu possa fazer para tornar as coisas melhores para mim. assim para sempre.

4. Não consigo imaginar que espécie de vida será a minha em dez anos.

5. Tenho tempo suficiente para realizar as coisas que quero fazer.

6. No futuro, eu espero ter sucesso no que mais me interessa.

7. Meu futuro me parece negro.

9. Simplesmente não consigo aproveitar as oportunidades e não há razão para que consiga, no futuro.

10. Minhas experiências passadas me prepararam bem para o futuro.

11. Tudo o que posso ver à minha frente é mais desprazer do que prazer.

12. Não espero conseguir o que realmente quero.

13. Quando penso no futuro, espero ser mais feliz do que sou agora.

14. As coisas simplesmente não se resolvem da maneira que eu quero.

15. Tenho uma grande fé no futuro.

16. Nunca consigo o que quero. Assim, é tolice querer qualquer coisa.

17. É pouco provável que eu vá obter qualquer satisfação real, no futuro.

18. O futuro me parece vago $\mathrm{e}$ incerto.

19. Posso esperar mais tempos bons do que maus.

20. Não adianta tentar realmente obter algo que quero, porque provavelmente não vou conseguir.

Coypright 01901 by NCS Pearesn. inc

Pearson coppright 01833 Aaron T. Eeck - Tracurago para a Lingua Portuguesa

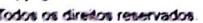

(P) 2017 Casapsl Livrarla e Editora Ltda

Eroticisa a reprosuras lotal ou parcial desta obra para qualquer finalidade Tosm os direilos recervasos. Avenista Frandisco Matarazo, 1500 - Conjunto 51 Estion Nhew York - Centro Empresanal Agua Branca Barra Funda. SAO PaubrSP . CEP OSO01-100 Tet (11) 3672-1240 - vrww pearsondinical com br

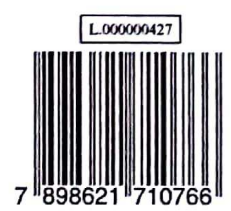


Anexo VII - BSI - Beck Scale for Suicide Ideation

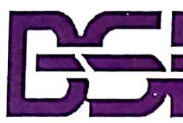

Data:

Nome: Estado Civil: Idade: Sexo:

Ocupação: Escolaridade:

Instruções: Por favor, leia cuidadosamente cada grupo de afirmações, abaixo. Faça um círculo na afirmação que em cada grupo melhor descreve como você tem se sentido na última semana, incluindo hoje. Tome o cuidado de ler todas as afirmações em cada grupo, antes de fazer uma escolha.

Parte 1

10 Tenho um desejo de viver que é de moderado a forte.

1 Tenho um desejo fraco de viver.

2 Não tenho desejo de viver.

20 Não tenho desejo de morrer.

2 Tenho um desejo de morrer que é de moderado a forte.

30 Minhas razões para viver pesam mais que minhas razões para morrer.

1 Minhas razões para viver ou morrer são aproximadamente iguais.

2 Minhas razões para morrer pesam mais que minhas razões para viver
1 Tenho um desejo fraco de morrer.

40 Não tenho desejo de me matar.

1 Tenho um desejo fraco de me matar.

2 Tenho um desejo de me matar que é de moderado a forte.

5 - Se estivesse numa situação de risco de vida, tentaria me salvar.

1 Se estivesse numa situação de risco de vida, deixaria vida ou morte ao acaso.

2 Se estivesse numa situação de risco de vida, não tomaria as medidas necessárias para evitar a morte.

Se você fez um círculo nas afirmações "zero", em ambos os grupos 4 e 5 , passe para o grupo 20 . Se você marcou "um" ou "dois", seja no grupo 4 ou 5, então abra a página e prossiga no grupo 6.

Subtotal da Parte 1

200 Nunca tentei suicídio.

1 Tentei suicídio uma vez.

2 Tentei suicídio duas ou mais vezes.

Se você tentou suicídio anteriormente, por favor, continue no próximo grupo de afirmações.

210 Durante a última tentativa de suicídio, meu desejo de morrer era fraco.

1 Durante a última tentativa de suicídio, meu desejo de morrer era moderado.

2 Durante a última tentativa de suicídio, meu desejo de morrer era forte.
Subtotal da Parte 2

\section{Escore Total}

"Traduzido e adaptado por permissão de The Psychological Corporation, U.S.A. Direitos reservados ${ }^{\circ} 1991$, a Aaron T. Beck.

Tradução para a língua portuguesa. Direitos reservados ${ }^{c} 1991$ a Aaron T. Beck. Todos os direitos reservados."

Tradução e adaptação brasileira, 2001, Casapsi ${ }^{\circledR}$ Livraria e Editora Ltda. BSI é um logotipo da Psychological Corporation. 


\section{Continuação BSI}

Parte 2

6 Tenho breves períodos com idéias de me matar que passam rapidamente.

Tenho períodos com idéias de me matar que duram algum tempo.

2 Tenho longos períodos com idéias de me matar.

7 - Raramente ou ocasionalmente penso em me matar

1 Tenho idéias freqüentes de me matar.

2 Penso constantemente em me matar.

8 Não aceito a idéia de me matar.

1 Não aceito, nem rejeito, a idéia de me matar.

2 Aceito a idéia de me matar.

90 Consigo me controlar quanto a cometer suicídio.

1 Não estou certo se consigo me controlar quanto a cometer suicídio.

2 Não consigo me controlar quanto a cometer suicídio.

10 Eu não me mataria por causa da minha família, de meus amigos, de minha religião, de um possivel dano por uma tentativa malsucedida etc.

1 Eu estou um tanto preocupado a respeito de me matar por causa da minha família, de meus amigos, de minha religião, de um possível dano por uma tentativa malsucedida etc.

2 Eu não estou ou estou só um pouco preocupado a respeito de me matar por causa da minha família, de meus amigos, de minha religião, de um possível dano por uma tentativa malsucedida etc.

11 Minhas razões para querer cometer suicídio têm em vista principalmente influenciar os outros, como conseguir me vingar das pessoas, torná-las mais felizes, fazê-las prestar mais atenção em mim etc.

1 Minhas razões para querer cometer suicídio não têm em vista apenas influenciar os outros, mas também representam uma maneira de solucionar meus problemas.

2 Minhas razões para querer cometer suicídio se baseiam principalmente numa fuga de meus problemas.

12 Não tenho plano específico sobre como me matar.

1 Tenho considerado maneiras de me matar, mas não elaborei detalhes.

2 Tenho um plano específico para me matar.
13 Não tenho acesso a um método ou uma oportunidade de me matar.

I O método que usaria para cometer suicídio leva tempo e realmente não tenho uma boa oportunidade de usá-lo.

2 Tenho ou espero ter acesso ao método que escolheria para me matar e, também, tenho ou teria oportunidade de usá-lo.

14 Não tenho a coragem ou a capacidade para cometer suicídio.

I Não estou certo se tenho a coragem ou a capacidade para cometer suicídio.

2 Tenho a coragem e a capacidade para cometer suicídio.

15 Não espero fazer uma tentativa de suicídio.

1 Não estou certo de que farei uma tentativa de suicidio.

2 Estou certo de que farei uma tentativa de suicídio.

16 Eu não fiz preparativos para cometer suicidio.

1 Tenho feito alguns preparativos para cometer suicídio.

2 Meus preparativos para cometer suicidio já estâo quase prontos ou completos.

17 Não escrevi um bilhete suicida.

I Tenho pensado em escrever um bilhete suicida ou comecei a escrever, mas não terminei.

2 Tenho um bilhete suicida pronto.

18 Não tomei providências em relação ao que acontecerá depois que eu tiver cometido suicidio.

1 Tenho pensado em tomar algumas providencias em relação ao que acontecerá depois que eu tiver cometido suicidio.

2 Tomei providencias definidas em relaça to ao que acontecerá depois que eu tiver cometido suicidio.

19 Não tenho escondido das pessoas o meu desejo de me matar.

1 Tenho evitado contar às pessoas sobre a vontade de me matar.

2 Tenho tentado nato revelar, esconder ou mentir sobre a vontade de cometer suicidio.

Passe para o Grupo 20.
20 - Nunca tentei suicídio.

1 Tentei suicídio uma vez.

2 Tentei suicídio duas ou mais vezes.

Se você tentou suicídio anteriormente, por favor, continue no próximo grupo de afirmações.

210 Durante a última tentativa de suicídio, meu desejo de morrer era fraco.

1 Durante a última tentativa de suicldio, meu descjo de morrer era moderado.

2 Durante a última tentativa de suicídio, meu desejo de morrer era forte.
Subtotal da Parte 2

Escore 'Total

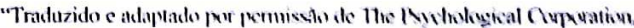

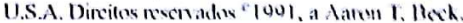

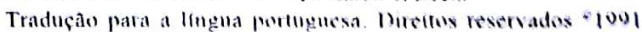

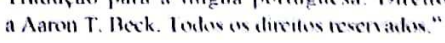

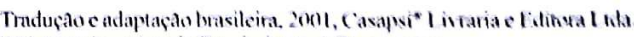
BSI e um logutipo da Psychelogical Conperation. 